\title{
On the determination of ruled and developable surfaces in Euclidean 3-space $E^{3}$
}

\author{
RASHAD A. ABDEL-BAKY ${ }^{1}$ and FATEMAH MOFARRE $H^{2}{ }^{*}$ \\ ${ }^{1}$ Department of Mathematics, Faculty of Science, University of Assiut, Assiut 71516, \\ Egypt \\ ${ }^{2}$ Mathematical Science Department, Faculty of Science, \\ Princess Nourah bint Abdulrahman University, Riyadh 11546, Saudi Arabia
}

\begin{abstract}
Depending on E. Study's map, the method of determination of ruled and developable surfaces is introduced. We show that dual vectorial expression of ruled and developable surfaces will be gained from coordinates and the first derivatives of the base curve. We illustrated this method by giving some representative examples.
\end{abstract}

Key-Words:Ruledanddevelopablesurfaces,Distributionparameter,E.Study'smap.

Received: April 13, 2020. Revised: October 14, 2020. Accepted: November 1, 2020. Published: November 15, 2020.

\section{Introduction}

In space, the movement of a straight line on the curve establishes ruled surface. These lines are said to be generators (rulings), and every curve that cuts all the generators is called a directrix (base curve). The theory of ruled surface has been highlighted by researchers as well mathematicians because it has many applications such as problems of designs in spatial mechanisms, physics, and kinematics [1-4]. Developable surfaces are special kinds of ruled surfaces, where any point of the same generator line share the common tangent plane. The rulings are principal curvature lines with vanishing normal curvature and the Gaussian curvature vanishes everywhere on the surface. As well known, the inner metric of a surface locates the Gaussian curvature, therefore all the lengths and angles on the surface remain invariant under bending. This advantage is what makes ruled and developable surfaces important in manufacturing. Hence, both ruled surfaces and developable surfaces have been paid attention in engineering, architecture, and design, etc. (See for example [1-9]).

Previously, mathematical techniques that relay on the so-called E. Study's map has been used to deal with ruled and developable surfaces in computer-aided geometric design (CAGD) [1015]. The E. Study's map concludes: All oriented lines set in Euclidean 3-space $E^{3}$ is in one-to- one correspondence with all points set of the dual unit sphere in the dual 3- space $D^{3}$. Specifically, more information of the dual elements and one-to-one correspondence between both ruled surfaces and the one-parameter dual spherical motions are in [1-4]. Some relevant studies can be found in [16], and $[17]$.

The present work is organized as follows: In section 2, basic definitions of the presentation of oriented lines in terms of dual vectors and $\mathrm{E}$ are introduced briefly. Study's map. In section 3, a method for determination of ruled surfaces is presented using dual vector calculus. It is also shown show that dual vectorial expression of the ruled surface is obtained from coordinates of the base curve. In terms of this, by using an alternative way than [14], The method for determination of developable ruled surfaces is constructed and the linear differential equation of first order is obtained. We illustrated the method by giving some representative examples.

\section{Basic concepts}

At this section some concepts, formulas of dual numbers and dual vectors are recalled [1-4]. Starting with the use of convenient line coordinates: The oriented in Euclidean 3 -space $E^{3}$ can be described using the points $\mathbf{p}$ and $\mathbf{q}$ as follow: in case $t$ is any constant that is not zero, the 
parametric equation of the line is:

$$
\mathbf{q}=\mathbf{p}+t \mathbf{x}, \mathbf{x}=\frac{\mathbf{q}-\mathbf{p}}{\|\mathbf{q}-\mathbf{p}\|},<\mathbf{x}, \mathbf{x}>\neq 0 .
$$

Then we define the moment of the vector $\mathbf{x}$ respecting to the fixed origin point in $E^{3}$ as

$$
\mathbf{x}^{*}=\mathbf{q} \times \mathbf{x}=\mathbf{p} \times \mathbf{x} .
$$

In other words, $\mathbf{x}^{*}$ is not changing for any choices of the points on the line. And the pair $\left(\mathbf{x}, \mathbf{x}^{*}\right) \in$ $E^{3} \times E^{3}$ satisfying the next two equations

$$
<\mathrm{x}, \mathrm{x}>=1,<\mathrm{x}, \mathrm{x}^{*}>=0 \text {. }
$$

The six components $x_{i}, x_{i}^{*}(i=1,2,3)$ of $\mathbf{x}$, and $\mathbf{x}^{*}$ named as normed Plucker coordinates of the line.

For the real numbers $x$ and $x^{*}$ the conjunction $X=x+\varepsilon x^{*}$ is called a dual number. In fact, $\varepsilon$ is the dual unit subject to the rules $\varepsilon \neq 0, \varepsilon^{2}=0, \varepsilon \cdot 1=1 . \varepsilon=\varepsilon$. The set of dual numbers, $D$, forms the commutative ring having the numbers $\varepsilon x^{*}\left(x^{*}\right.$ real $)$ as divisors of zero, not a field. At algebra there is no inverse of $\varepsilon a^{*}$. However, other rules of algebra of dual numbers are similar to the rules at the complex numbers. Employing matrices, dual numbers may be presented by

$\varepsilon=\left(\begin{array}{cc}0 & 1 \\ 0 & 0\end{array}\right)$, and $x+\varepsilon x^{*}=\left(\begin{array}{cc}x & x^{*} \\ 0 & x\end{array}\right)$

The sum and product of dual numbers is similar to adding or multiplying matrices; they are also commutative and associative which is corresponding to normal matrix presentation of complex numbers [1-4].

For all pairs $\left(\mathbf{x}, \mathbf{x}^{*}\right) \in E^{3} \times E^{3}$ the set

$$
D^{3}=\left\{\mathbf{X}=\mathbf{x}+\varepsilon \mathbf{x}^{*}, \varepsilon \neq 0, \varepsilon^{2}=0\right\},
$$

consider it in addition to scalar product

$$
\begin{aligned}
<\mathbf{X}, \mathbf{Y}>= & <\mathbf{x}, \mathbf{y}> \\
& +\varepsilon\left(<\mathbf{y}, \mathbf{x}^{*}>+<\mathbf{y}^{*}, \mathbf{x}>\right),
\end{aligned}
$$

forms the dual 3-space $D^{3}$. Therefore, the point $\mathbf{X}=\left(X_{1}, X_{2}, X_{3}\right)^{t}$ has dual coordinates $X_{i}=$ $\left(x_{i}+\varepsilon x_{i}^{*}\right) \in D$. The norm is defined as

$$
<\mathbf{X}, \mathbf{X}>^{\frac{1}{2}}:=\|\mathbf{X}\|=\|\mathbf{x}\|\left(1+\varepsilon \frac{<\mathbf{x}, \mathbf{x}^{*}>}{\|\mathbf{x}\|^{2}}\right) .
$$

In the dual 3 -space $D^{3}$ the dual unit sphere is defined as

$$
K=\left\{\mathbf{X} \in D^{3} \mid\|\mathbf{X}\|^{2}=X_{1}^{2}+X_{2}^{2}+X_{3}^{2}=1\right\} .
$$

Definition of dual unit sphere gives us that all points $\mathbf{X}$ of $K$ must fluffily two equations

$$
x_{1}^{2}+x_{2}^{2}+x_{3}^{2}=1, \quad x_{1} x_{1}^{*}+x_{2} x_{2}^{*}+x_{3} x_{3}^{*}=0 .
$$

Through this paper we have the following map ( [1-4] (E. Study's map): The set of all oriented lines in Euclidean $E^{3}$ is in one-to-one correspondence with the set of points of dual unit sphere in the dual 3 -space $D^{3}$. The exemplification of directed lines in $E^{3}$ by dual unit vectors results in various advantages and from this part we will not distinguish between directed lines and their corresponding dual unit vectors.

\subsection{Ruled and developable surfaces}

A ruled surface is the one-parameter set of straight lines. Similar surface has the parameterization in the ruled form:

$$
M: \mathbf{r}(t, v)=\mathbf{y}(t)+v \mathbf{x}(t), v \in R,
$$

where $\mathbf{y}=\mathbf{y}(t)$ is its base curve, and $\mathbf{x}=\mathbf{x}(t)$ is the unit vector giving the direction of the straight lines (rulings) of the surface. The E. Study map leads to rewriting Eq. (9) using dual vector function as

$$
M: \mathbf{X}(t)=\mathbf{x}(t)+\varepsilon \mathbf{y}(t) \times \mathbf{x} t)=\mathbf{x}(t)+\varepsilon \mathbf{x}^{*}(t),
$$

because of the spherical image $\mathbf{x}$ that is a unit vector, the dual vector $\mathbf{X}$ has unit magnitude as is seen from the calculation

$$
\begin{aligned}
\|\mathbf{X}\|^{2} & =\langle\mathbf{x}+\varepsilon \mathbf{y} \times \mathbf{x}, \mathbf{x}+\varepsilon \mathbf{y} \times \mathbf{x}> \\
= & \|\mathbf{x}\|^{2}+2 \varepsilon<\mathbf{x}, \mathbf{y} \times \mathbf{x}> \\
& \quad+\varepsilon^{2}<\mathbf{y} \times \mathbf{x}, \mathbf{y} \times \mathbf{x}> \\
= & \|\mathbf{x}\|^{2}=1 .
\end{aligned}
$$

The dual arc length of $\mathbf{X}(t)$ is defined as

$$
\begin{aligned}
d S & =d s+\varepsilon d s^{*}=\left\|\mathbf{x}^{\prime}\right\| d t \\
& =\left\|\mathbf{x}^{\prime}\right\|\left(1+\varepsilon \frac{<\mathbf{x}^{\prime}, \mathbf{x}^{*^{\prime}}>}{\left\|\mathbf{x}^{\prime}\right\|^{2}}\right) d t .
\end{aligned}
$$

where a dash denotes differentiation with respect to $t$. Hence, the distribution parameter is given by

$$
\lambda(t):=\frac{d s^{*}}{d s}=\frac{<\mathbf{x}^{\prime}, \mathbf{x}^{*^{\prime}}>}{\left\|\mathbf{x}^{\prime}\right\|^{2}} .
$$

In Eq. (12): (a) in case $\lambda(t)=0$, therefore $M$ is the developable ruled surface (b) if $\mathbf{x}^{\prime}=\mathbf{0}$, then $M$ is the cylindrical surface. 


\section{The determination of ruled and developable surfaces}

We develop the method to construct ruled and developable surfaces by the use of dual vector calculus. The dual coordinates $X_{i}=\left(x_{i}+\varepsilon x_{i}^{*}\right)$ of an arbitrary point $\mathbf{X}$ of the dual unit sphere $K$ can be given by:

$$
\mathbf{X}=(\cos \Phi, \sin \Phi \cos \Psi, \sin \Phi \sin \Psi) .
$$

where $\Theta=\vartheta+\varepsilon \vartheta^{*}$, and $\Psi=\psi+\varepsilon \psi^{*}$ are dual angles. Furthermore, considering $\mathbf{X}=\mathbf{X}(t), t \in R$ that is corresponds to the ruled surface $M$.Then, the dual arc-length of $\mathbf{X}(t)$ is given by

$$
d S:=d s+\varepsilon d s^{*}=\sqrt{\Psi^{\prime 2} \sin ^{2} \Phi+\Phi^{\prime 2}} d t .
$$

Dividing real and the dual parts of Eq. (14), in the same order, we have:

$$
\mathrm{d} s=\sqrt{\psi^{\prime 2} \sin ^{2} \varphi+\varphi^{\prime 2}} d t,
$$

and

$$
\mathrm{ds}^{*}=\frac{\psi^{\prime} \psi^{*^{\prime}} \sin ^{2} \varphi+\varphi^{*} \psi^{\prime 2} \sin \varphi \cos \varphi+\varphi^{\prime} \varphi^{*^{\prime}}}{\sqrt{\psi^{\prime 2} \sin ^{2} \varphi+\varphi^{\prime 2}}} .
$$

Hence we arrive at

$$
\begin{gathered}
\lambda(t):=\frac{d s^{*}}{d s} \\
=\frac{\psi^{\prime} \psi^{*^{\prime}} \sin ^{2} \varphi+\varphi^{*} \psi^{\prime} \sin \varphi \cos \varphi+\varphi^{\prime} \varphi^{*^{\prime}}}{\psi^{\prime 2} \sin ^{2} \varphi+\varphi^{\prime 2}} .
\end{gathered}
$$

It is obvious that if $\psi(t)$, and $\varphi(t)$ are both constants, that is $\left\|\mathbf{x}^{\prime}\right\|=0$, then $M$ is a cylinder in $E^{3}$.

Since $\varepsilon^{2}=\varepsilon^{3}=\ldots=0$, the Plucker coordinates of $\mathbf{X}$ are:

$$
\left.\begin{array}{rl}
x_{1}=\cos \varphi, x_{1}^{*}=-\varphi^{*} \sin \varphi \\
x_{2}=\sin \varphi \cos \psi, x_{2}^{*} & =\varphi^{*} \cos \varphi \cos \psi \\
& -\psi^{*} \sin \psi \sin \varphi, \\
x_{3}=\sin \varphi \sin \psi, x_{3}^{*} & =\varphi^{*} \cos \varphi \sin \psi \\
& +\psi^{*} \cos \psi \sin \varphi .
\end{array}\right\}
$$

Now, it seems natural to pose the following question: when we are given a curve $\mathbf{y}(t)=$ $\left(y_{1}(t), y_{2}(t), y_{3}(t)\right)$ can we define the ruled surface where its base curve is the curve $\mathbf{y}(t)$ ?. The answer is affirmative and can be stated as follows: Since $\mathbf{x}^{*}=\mathbf{y} \times \mathbf{x}$ we have the system of linear equations in $y_{i}$ for $\mathrm{i}=1,2,3$ ( $y_{i s}$ are the coordinates of $\mathbf{y}$ ):

$$
\left.\begin{array}{c}
y_{2} \sin \varphi \sin \psi-y_{3} \sin \varphi \cos \psi=x_{1}^{*}, \\
-y_{1} \sin \varphi \sin \psi+y_{3} \cos \varphi=x_{2}^{*}, \\
y_{1} \sin \varphi \cos \psi-y_{2} \cos \varphi=x_{3}^{*} .
\end{array}\right\}
$$

The matrix of coefficients of unknowns $y_{1}, y_{2}$, and $y_{3}$ is the skew symmetric matrix

$$
\left(\begin{array}{ccc}
0 & \sin \varphi \sin \psi & -\sin \varphi \cos \psi \\
-\sin \varphi \sin \psi & 0 & \cos \varphi \\
\sin \varphi \cos \psi & -\cos \varphi & 0
\end{array}\right)
$$

and thus its rank is 2 with $\varphi(t) \neq 2 \pi k$ ( $k$ is an integer). The rank of the augmented matrix

$$
\left(\begin{array}{cccc}
0 & \sin \varphi \sin \psi & -\sin \varphi \cos \psi & x_{1}^{*} \\
-\sin \varphi \sin \psi & 0 & \cos \varphi & x_{2}^{*} \\
\sin \varphi \cos \psi & -\cos \varphi & 0 & x_{2}^{*}
\end{array}\right)
$$

is also 2. Thereby, this system has infinite solutions given by

$$
\begin{gathered}
y_{2}=\left(y_{1}-\psi^{*}\right) \tan \varphi \cos \psi-\varphi^{*} \sin \psi, \\
y_{3}=\left(y_{1}-\psi^{*}\right) \tan \varphi \sin \psi+\varphi^{*} \cos \psi, \\
y_{1}=y_{1}(\vartheta(t), \varphi(t)) .
\end{gathered}
$$

Since $y_{1}(t)$ can be chosen arbitrarily, then we may take $y_{1}(t)=\psi^{*}(t)$. In this case, Eqs. (18) reduces to

$$
y_{1}(t)=\psi^{*}, y_{2}(t)=-\varphi^{*} \sin \psi, y_{3}(t)=\varphi^{*} \cos \psi .
$$

From Eq. (19), we have

$$
y_{1}(t)=\psi^{*}, \varphi^{*}(t)=\mp \sqrt{y_{2}^{2}+y_{3}^{2}}, \tan \psi=-\frac{y_{2}}{y_{3}} .
$$

Notably, $\varphi^{*}(t)$ has two values; using minus sign results in reciprocal of the ruled surface obtained by the use of plus sign. Therefore through the paper we choice lower sign. Into Eq. (9) we substitute from Eqs. (19), (20) and obtain:

$$
\begin{gathered}
\mathbf{r}(t, v)=\left(y_{1}, y_{2}, y_{3}\right)_{2} \\
+v\left(\cos \varphi, \frac{y_{3}}{\sqrt{y_{2}^{2}+y_{3}^{2}}} \sin \varphi,-\frac{y_{2}}{\sqrt{y_{2}^{2}+y_{3}^{2}}} \sin \varphi\right),
\end{gathered}
$$

where $y_{2}^{2}+y_{3}^{2} \neq 0, v \in R$, and $\varphi(t)$ is arbitrary.

Theorem 1. Suppose $\mathbf{y}(t)$ is the regular curve in Euclidean 3 -space $E^{3}$. Therefore there is a family of ruled surface represented by Eq. (21).

Example 1. Let $\mathbf{y}(t)=\left(2 t+1, t^{2}, t^{2}\right)$ be a curve in Euclidean 3-space $E^{3}$. Then, in view of Eq. (21), the family of ruled surface is

$$
\mathbf{r}(t, v)=\left(2 t+1, t^{2}, t^{2}\right)
$$




$$
+v\left(\cos \varphi, \frac{t}{\sqrt{2}} \sin \varphi,-\frac{t}{\sqrt{2}} \sin \varphi\right) v \in R .
$$

The distribution parameter is $\lambda(t)=\frac{\sqrt{2}}{\varphi^{\prime}}$. The function $\varphi(t)$ can control the shape of the surface. In case $\varphi(t)=t$, then $\lambda(t)=\sqrt{2}$ for $-\pi \leq t \leq \pi$, and $-10 \leq v \leq 10$, the ruled surface is shown in Figure. 1. If $\vartheta(t)=-t$, then $\lambda(t)=-\sqrt{2}$, $-\pi \leq t \leq \pi$, and $-10 \leq v \leq 10$ the surface is shown in Figure 2.

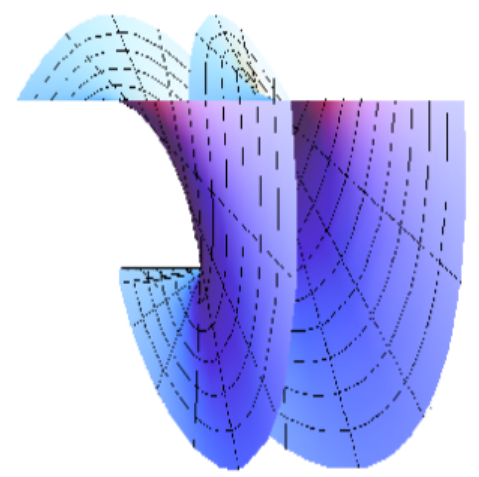

Figure 1:

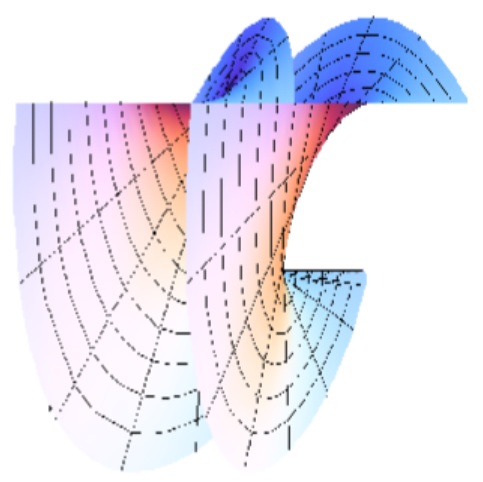

Figure 2:

Example 2. Given the cylindrical helix $\mathbf{y}(t)=\left(t^{3} / 3,2 t, t^{2} / 2\right)$. Likewise, we have:

$$
\begin{array}{cc}
\mathbf{r}(t, v)= & \left(t^{3} / 3,2 t, t^{2} / 2\right) \\
+ & v\left(\cos \varphi, \frac{4 t}{\sqrt{t^{2}+16}} \sin \varphi,-\frac{4 t}{\sqrt{t^{2}+16}} \sin \varphi\right)
\end{array}
$$

The distribution parameter is

$$
\lambda(t)=\sqrt{t^{2}+16} \Omega
$$

where

$$
\Omega=\left(\frac{4 t^{2} \sqrt{t^{2}+16} \sin ^{2} \varphi+4 \sin 2 \varphi+\left(t^{2}+8\right)\left(t^{2}+16\right) \varphi^{\prime}}{16 \sin ^{2} \varphi+\left(t^{2}+16\right) \varphi^{\prime 2}}\right) .
$$

If we take

$$
\varphi(t)=t
$$

then for $-\pi \leq t \leq \pi,-10 \leq v \leq 10$, the ruled surface is shown in Fig. 3, and the distribution parameter is

$$
\lambda(t)=\sqrt{t^{2}+16} \Omega_{1}
$$

where

$\Omega_{1}=\left(\frac{4 t^{2} \sqrt{t^{2}+16} \sin ^{2} t+4 \sin 2 t+\left(t^{2}+8\right)\left(t^{2}+16\right)}{16 \sin ^{2} t+\left(t^{2}+16\right)}\right)$.

For $\varphi(t)=-t, \pi \leq t \leq \pi,-10 \leq v \leq 10$ the surface is shown in Figure 4, and the distribution parameter is

$$
\lambda(t)=\sqrt{t^{2}+16} \Omega_{2}
$$

where

$$
\Omega_{2}=\left(\frac{4 t^{2} \sqrt{t^{2}+16} \sin ^{2} t-4 \sin 2 t-\left(t^{2}+8\right)\left(t^{2}+16\right)}{16 \sin ^{2} t+\left(t^{2}+16\right)}\right) .
$$




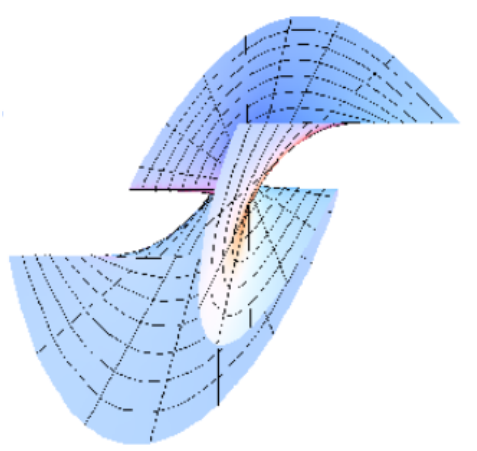

Figure 4:

The coming is the alternative way to discuss the notions of developable surfaces discussed in [14]. Under what condition $\mathbf{y}(t, v)$ is a developable ruled surface in Euclidean 3 -space $E^{3}$ ?. The answer is positive and can be stated as follows: In fact, in view of Eq. $(15), \mathbf{y}(t, v)$ is developable if and only if $\lambda(t)=0$, that is,

$$
\psi^{\prime} \psi^{*^{\prime}} \sin ^{2} \varphi+\varphi^{*} \psi^{\prime 2} \sin \varphi \cos \varphi+\varphi^{\prime} \varphi^{*^{\prime}}=0 .
$$

From Eq. (24), we get

$$
(\cot \varphi)^{\prime}+\frac{\varphi^{*} \psi^{\prime 2}}{\varphi^{*^{\prime}}} \cot \varphi+\frac{\psi^{\prime} \psi^{*^{\prime}}}{\varphi^{*^{\prime}}}=0 .
$$

Setting

$$
f(t)=\cot \varphi, G(t)=\frac{\varphi^{*} \psi^{\prime 2}}{\varphi^{*^{\prime}}}, H(t)=\frac{\psi^{\prime} \psi^{*^{\prime}}}{\varphi^{*^{\prime}}},
$$

we are lead to a linear differential equation of first order

$$
\frac{d f(t)}{d t}+G(t) f(t)+H(t)=0 .
$$

The determination of $\varphi(t)$ is needed here. The solution of (26) results in $\cot \varphi$ which contains the integral constant, therefore there are infinitely developable ruled surfaces where every one has the base curve $\mathbf{y}(t)$.

Example 3. By making use of the curve in example 1 , it is easy to show that

$$
\tan \psi=-1, \varphi^{*}=t \sqrt{2}, \psi^{*}=2 t+1
$$

and

$$
\psi^{*^{\prime}}=\sqrt{2}, \psi^{\prime}=0, \varphi^{*^{\prime}}=\sqrt{2},
$$

we substitute these values into Eq. (26) and solve this differential equation

$$
f(t)=\cot \varphi=c, c \in R .
$$

Since $\cot \varphi=c$, then we have:

$$
\cos \varphi= \pm \frac{c}{\sqrt{1+c^{2}}}, \sin \varphi= \pm \frac{1}{\sqrt{1+c^{2}}} .
$$

Choosing the plus sign, the family of developable ruled surface can be written as

$$
\mathbf{r}(t, v)=\left(2 t+1, t^{2}, t^{2}\right)+\frac{v}{\sqrt{1+c^{2}}}(c, 1,-1),
$$

where $v \in R$. If $c=1,-2 \leq t \leq 2$, and $-3 \leq v \leq 3$, therefore the member of this family is obtained as in Figure 5. Figure 6 shows the surface with $c=-1,-2 \leq t \leq .2$, and $-3 \leq v \leq 3$.

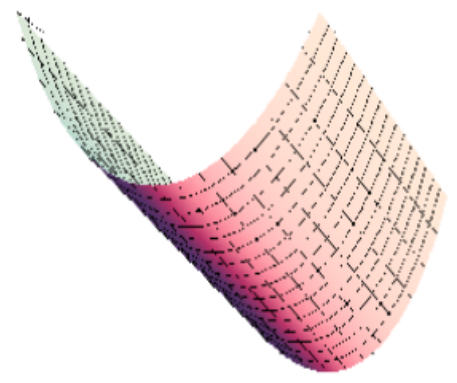

Figure 5:

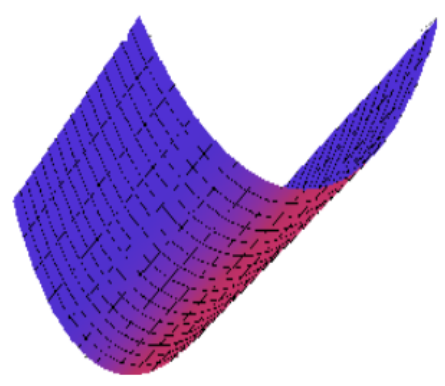

Figure 6:

Example 4. Form the curve in example 2, we have

$$
\tan \psi=-\frac{4}{t}, \varphi^{*}=\frac{t}{2} \sqrt{16+t^{2}}, \psi^{*}=\frac{t^{3}}{3} .
$$

Then

$$
\begin{gathered}
\psi^{*^{\prime}}=t^{2}, \psi^{\prime}=\frac{4}{16+t^{2}}, \varphi^{*^{\prime}}=\frac{8+t^{2}}{\sqrt{16+t^{2}}}, \\
G(t)=\frac{8 t}{\left(16+t^{2}\right)\left(8+t^{2}\right)}, H(t)=-\frac{4 t^{2} \sqrt{16+t^{2}}}{\left(16+t^{2}\right)\left(8+t^{2}\right)} .
\end{gathered}
$$


According to Eqs (26), and (29), we have:

$$
\frac{d f(t)}{d t}+\frac{8 t}{\left(16+t^{2}\right)\left(8+t^{2}\right)} f(t)=\frac{4 t^{2} \sqrt{16+t^{2}}}{\left(16+t^{2}\right)\left(8+t^{2}\right)} .
$$

The solution of this differential equation gives

$$
f(t)=-4 \sqrt{\frac{8+t^{2}}{16+t^{2}}} K
$$

where $K=\left[\ln \left(t+\sqrt{8+t^{2}}\right)+\frac{8}{8+t^{2}+t \sqrt{8+t^{2}}}+c\right]$, $c \in R$. Since $f(t)=\cot \varphi$, then we have:

$$
\sin \varphi= \pm \frac{1}{\sqrt{1+f^{2}(t)}}, \cos \varphi= \pm \frac{f(t)}{\sqrt{1+f^{2}(t)}}
$$

Hence, the family of the developable ruled surface is given by

$$
\begin{aligned}
\mathbf{r}(t, v)= & \left(t^{3} / 3,2 t, t^{2} / 2\right) \\
& +\frac{v}{\sqrt{1+f^{2}(t)}}\left(f(t), \frac{t}{\sqrt{2}},-\frac{t}{\sqrt{2}}\right),
\end{aligned}
$$

where $v \in R$. If $\mathrm{c}=1,-2 \leq t \leq 2$, and $-4 \leq v \leq 4$, therefore the member of this family is obtained as in Figure 7. Figure 8 shows the surface with $c=-1,-1 \leq t \leq 1$, and $-2 \leq v \leq-2$.

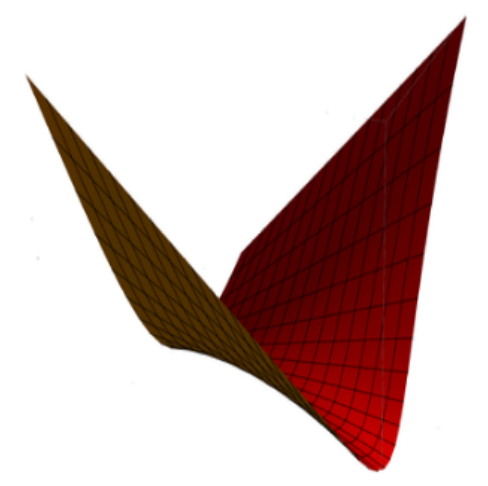

Figure 7:

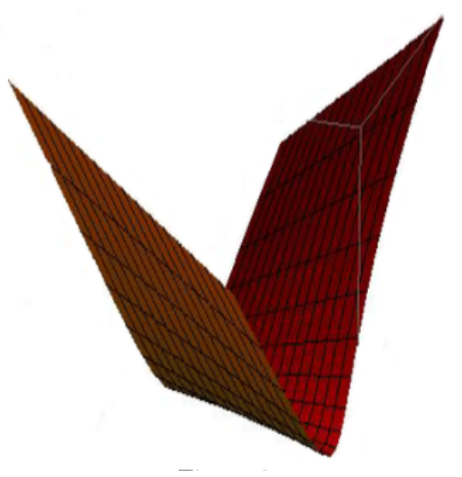

Figure 8:

\section{Conclusion}

The use of some mathematical methods in E. Study map has been found suitable for the determination of ruled and developable surfaces. Similar results are used widely in different fields such as in sheet and plate metal industry [5], in boat hulls design, the design of windshield and as binder (blank holder) surfaces for sheet.

\section{Acknowledgements}

This research was funded by the Deanship of Scientific Research at Princess Nourah bint Abdulrahman University through the Fast-track Research Funding Program. The authors gratefully acknowledge the reviewers whose comments assisted in improving this work.

\section{References:}

[1] O. Bottema, and B. Roth. Theoretical Kinematics, North-Holland Press, New York 1979.

[2] A. Karger, and J. Novak. Space Kinematics and Lie Groups, Gordon and Breach Science Publishers, New York 1985.

[3] J.M. McCarthy. On the scalar and dual formulations of the curvature theory of line trajectories. ASME J. of Mech., Trans and Auto. in Design,1987, 101-106.

[4] H. Pottman, and J. Wallner. Computational Line Geometry, Springer-Verlag, Berlin, Heidelberg 2001.

[5] M.J. Mancewicz, and W.H. Frey. Developable Surfaces: Properties, Representations and Methods of Design, General Motors R\&D Publication, 1992. 
[6] R.M.C Bodduluri, B. Ravani, Design of developable surfaces using duality between plane and point geometries, Computer-Aided Design, Vol.25, No.10, 1993, pp. 621-632.

[7] H.Y. Zhao , and G.J. Wang. A new method for designing a developable surface utilizing the surface pencil through a given curve, Prog. Nat. Sci. Vol.18, No.1, 2008, pp. 105-110.

[8] C.Y. Li, R.H. Wang, and C.G. Zhu. An approach for designing a developable surface through a given line of curvature, ComputerAided Design, Vol.45, 2013, pp. 621-627.

[9] Jianmin Wang, Ping Jiang, Yu Guo, and Jing Meng. Developable surface pencil pairs with special pairs as common asymptotes, $A M C$, Vol. 362, 2019, 124583.

[10] O. Kose. Contributions to the theory of integral invariants of a closed ruled surface. Mech. and Mach. Theory, Vol. 32, No.2, 1997, pp. 261-277.

[11] R.A. Abdel-Baky, and F.R. Al-Solamy. A new geometrical approach to one-parameter spatial motion, J. of. Eng. Maths, Vol. 60, 2008, pp. 149-172.

[12] R.A. Abdel-Baky, and R.A Al-Ghefari. On the one-parameter dual spherical motions, Computer Aided Geometric Design, Vol. 28, 2011, pp. 23-37.

[13] R.A Al-Ghefari, and R.A. Abdel-Baky. Kinematic geometry of a line trajectory in spatial motion, J. of Mechanical Science and Technology, Vol. 29, No. 9, 2015, pp. 35973608 .
[14] O. Kose. A method of the determination of a developable ruled surface. Mech. Mach. Theory,Vol. 34, 1999, pp. 1187-1193.

[15] O.G Yıldız, S.O. Karakus, and H.H. Hacisalihoglu. On the determination of a developable spherical orthotomic ruled surface, Bull. Math. Sci. Vol. 5, 2015, pp.137-146.

[16] Libor Pekar. On Simple Algebraic Control Design and Possible Controller Tuning for Linear Systems with Delays, Int. J. of Circuits, Systems and Signal Processing,Vol.12, 2018, pp. 178-191.

[17] Chengfang Tan, Caiyin Wang, Yulin Li, and Xixi Qi. Recommended Items Rating Prediction based on RBF Neural Network Optimized by PSO Algorithm, Int. J. of Circuits, Systems and Signal Processing, Vol.12, 2018, pp. 580-586.

Follow: www.wseas.org/multimedia/contributorrole-instruction.pdf

\section{Creative Commons Attribution License 4.0 (Attribution 4.0 International, CC BY 4.0)}

This article is published under the terms of the Creative Commons Attribution License 4.0 https://creativecommons.org/licenses/by/4.0/deed.en_US 